\title{
Regulation of prostate cancer immunity-related genes in PC3 prostate cancer cells by ETS-1
}

\author{
ZAKI SHAIKHIBRAHIM, ANDREAS LINDSTROT, REINHARD BUETTNER and NICOLAS WERNERT
}

Institute of Pathology, University Hospital Bonn, D-53127 Bonn, Germany

Received August 30, 2011; Accepted November 21, 2011

DOI: 10.3892/ol.2011.509

\begin{abstract}
Prostate cancer (PCa) is one of the most prevalent forms of cancer affecting males worldwide, and knowledge of the immune defenses involved in $\mathrm{PCa}$ remains incomplete. Since the identification of immunity-related genes may have enormous implications for the understanding of $\mathrm{PCa}$ immunology, we recently reported the identification of immunity-related genes in PCa tissues and found potential binding sites for the ETS family prototype, ETS-1, in the majority of genes identified. Therefore, as a continuation of our previous study, we investigated whether ETS-1 regulates these genes in an in vitro $\mathrm{PCa}$ cell line model, PC3 cells. We specifically blocked ETS-1 in PC3 cells by transfection with an ETS-1 inverse antisense expression vector or a mock control vector. We then assessed the effect of the blockade on the expression of the recently identified PCa immunity-related genes using a comprehensive oligo gene expression microarray analysis. The results showed that ETS-1 is involved in the activation or repression of the recently identified immunity-related genes in $\mathrm{PCa}$. These findings provide insights into the regulation of immunity-related genes in $\mathrm{PCa}$, and emphasize the importance of ETS-1 in prostate cancer immunology.
\end{abstract}

\section{Introduction}

Prostate cancer (PCa) is one of the most prevalent forms of cancer affecting males, and a leading cause of cancer mortality (1). The involvement of the immune response in tumor progression and disease outcome remains a matter of debate (2) and, in particular, knowledge of the immune defenses involved in PCa is still lacking. The association between the immune system of the body and tumor behavior has been reported (2), along with indications that the immune system may promote and inhibit tumor growth (2). For instance, the

Correspondence to: Professor Nicolas Wernert, Institute of Pathology, University Hospital Bonn, 25 Sigmund-Freud-Str., D-53127 Bonn, Germany

E-mail: nicolas.wernert@ukb.uni-bonn.de

Key words: ETS-1, transcriptional regulation, immunity, prostate cancer majority of pro-inflammatory cytokines generated by immune cells of the host or tumor cells promote tumor development, while pro-apoptotic and anti-inflammatory cytokines mostly interfere with tumor development (3).

In PCa, reduced infiltration of tumor-associated macrophages and absent or weak tumor infiltrating lymphocytes have been reported to be associated with tumor progression $(4,5)$. The majority of patients with $\mathrm{PCa}$ are immunocompetent, even though the host immunity is altered owing to tumor avoidance mechanisms (6). However, the lack of action of the immune defenses against the tumor may indicate an immunological tolerance (6). Moreover, a combination of Th1-adaptive immunity and inflammation genes has recently been suggested to confer improved prognosis of PCa (2).

Since the identification of immunity-related genes may have large implications for the understanding of PCa immunology, we recently reported the identification of immunity-related genes (B-cell, innate and T-cell immunity) in $\mathrm{PCa}$, and reported on their potential regulation by members of the ETS family of transcription factors (7). Of note, our analysis of the promoter regions of the identified immunity-related genes revealed that 31 out of the 37 genes have potential binding sites for the ETS family prototype, ETS-1 (7). In the majority of PCa cases, recurrent gene fusions involving members of the ETS family have frequently been reported (8), along with reports suggesting that members of the family are essential for the regulation of the immune system and immunity-related genes (9).

ETS-1, the prototype of the ETS family, has been reported to be overexpressed in latent and clinically manifest $\mathrm{PCa}$, and poor tumor differentiation has been associated with strong ETS-1 expression (10). Moreover, we have, in previous studies, reported that ETS-1 promotes proliferation, migration and/or invasion of various neoplastic cells, including melanoma, HeLa, glioma and PC3 prostate cells, and plays a role in the regulation of genes involved in the metastatic cascade (11-15).

As a continuation of our recent study, in which we identified immunity-related genes in PCa and reported on their potential regulation by ETS-1 (7), we investigated whether ETS-1 regulates these immunity-related genes in PC3 PCa cells.

Therefore, we specifically blocked ETS -1 in PC3 PCa cells and assessed the effect of the blockade on the expression of the recently identified PCa immunity-related genes using a comprehensive oligo gene expression microarray 
analysis. Results showed that 8 of the recently identified $\mathrm{PCa}$ immunity-related genes are regulated by ETS-1 in PC3 PCa cells. These findings provide insights into the regulation of immunity-related genes in PCa and emphasize the importance of ETS-1 in PCa immunology.

\section{Materials and methods}

Cell culture. The PCa cell line PC3 cells were grown in F12K media with L-glutamine (Invitrogen, Carlsbad, CA, USA) supplemented with penicillin, streptomycin (Invitrogen) and $10 \%$ heat-inactivated fetal calf serum (Invitrogen). In addition, $4.3 \mathrm{mg} / \mathrm{ml}$ of G418 (PAA Laboratories $\mathrm{GmbH}$, Pasching, Austria) was added to the media along with the transfected cells.

Blockade of ETS-1 in the PC3 PCa cell line. Using the calcium phosphate method as described previously (16), the plasmids pcDNA3.1h-ets-1 inverse and pcDNA3.1 (-) (mock control) were transfected into the PC3 cells. After $24 \mathrm{~h}$, selection was initiated with $4.3 \mathrm{mg} / \mathrm{ml}$ of G418 (PAA Laboratories $\mathrm{GmbH}$ ). Cells were lysed by freezing and thawing at room temperature, and a total of $20 \mu \mathrm{g}$ of protein was analysed on $10 \%$ sodium dodecyl sulfate (SDS)-polyacrylamide gel electrophoresis, and transferred onto nitrocellulose filters (Bio-Rad, Hercules, CA, USA). The nitrocellulose filters were then blocked with $5 \%$ dry milk in TBST (50 mM Tris, $150 \mathrm{mM} \mathrm{NaCl}, 0.1 \%$ Tween-20, pH 7.5), incubated in TBST with anti-Ets-1 mouse monoclonal antibodies (1:5000; Transduction Laboratories), and finally with anti-mouse $\mathrm{IgG}$ horse radish peroxidase-conjugated secondary antibody (1:5000; Amersham Biosciences, Piscataway, NJ, USA). For internal control, the housekeeping protein $\beta$-actin was evaluated using the mouse monoclonal anti- $\beta$-actin antibody (1:5000; Sigma, St Louis, MO, USA). The antibodies were then detected using enhanced chemiluminescent (ECL) reagents (Amersham Biosciences).

Whole human genome oligo microarray analysis of RNA isolated from PC3 cells with ETS-1 blockade and mock control. Using the RNeasy Mini kit (Qiagen, Hilden, Germany), RNA was isolated from PC3 cells with ETS-1 blockade and mock control according to the manufacturer's instructions. The recommended DNase digestion was performed with the RNase-Free DNase set (Qiagen). The amount of RNA was then measured using the ND1000 Nanodrop (Thermo Fisher Scientific, Waltham, MA, USA). The RNA was then sent to Miltenyi Biotech (Bergisch Gladbach, Germany), for whole human genome oligo microarray analysis and bioinformatical interpretation. The RNA was labeled with Cy3 and hybridized on the Whole Human Genome Oligo Microarray 4x44K (Agilent, Santa Clara, CA, USA) according to the manufacturer's instructions. The results of the entire microarrays were validated by examining the expression of a subset of genes using quantitative reverse transcriptase-polymerase chain reaction (qRT-PCR) analysis.

\section{Results}

ETS-1 blockade in PC3 PCa cells. Western blot analysis of cell lysates prepared from two stable PC3 cell cultures that
Table I. Genes that are upregulated $>2$-fold.

\begin{tabular}{llc}
\hline Gene name & \multicolumn{1}{c}{ Long name } & Fold-change \\
\hline CD38 & Cd38 molecule & 2.60 \\
HDAC9 & Histone deacetylase 9 & 7.24 \\
\hline
\end{tabular}

$\mathrm{P}<0.01$ denotes statistically significant differences in PC3 cells with ETS- 1 blockade compared to mock control cells.

Table II. Genes that are downregulated $>2$-fold.

\begin{tabular}{|c|c|c|}
\hline Gene name & Long name & Fold-change \\
\hline$B C L 11 A$ & B-cell CLL/lymphoma 11A & -2.77 \\
\hline CRISP3 & $\begin{array}{l}\text { Cysteine-rich secretory } \\
\text { protein } 3\end{array}$ & -5.12 \\
\hline$D M B T 1$ & $\begin{array}{l}\text { Deleted in malignant } \\
\text { brain tumors } 1\end{array}$ & -18.36 \\
\hline$F G R$ & $\begin{array}{l}\text { Gardner-Rasheed feline } \\
\text { sarcoma viral (v-fgr) } \\
\text { oncogene homolog }\end{array}$ & -2.32 \\
\hline NOS2 & $\begin{array}{l}\text { Nitric oxide synthase } 2 \text {, } \\
\text { inducable }\end{array}$ & -2.19 \\
\hline$S A A 1$ & $\begin{array}{l}\text { Serum amyloid A1, } \\
\text { transcript variant } 1\end{array}$ & -7.71 \\
\hline
\end{tabular}

A p-value $<0.01$ denotes statistically significant differences in PC3 cells with ETS-1 blockade compared to mock control cells.

were established by transfection with an ETS-1 inverse antisense expression vector or a mock control vector revealed the absence of ETS-1 in the inverse-transfected cells compared to the presence of ETS-1 in the mock control transfected cells over a period of 121 days (14).

Gene expression analysis of previously reported PCa immunity-related genes in PC3 cells with ETS-1 blockade compared to mock control. We have recently reported the identification of 37 immunity-related genes (B cell, innate and T-cell immunity) in PCa tissues, and found binding sites for ETS-1 within the promoter regions of 31 out of 37 of these genes (7). Therefore, the recently identified immunity-related genes were verified for their presence in the results of the gene expression microarray analysis of PC3 cells with ETS- 1 blockade compared to mock control. Only genes with an expression of $>2$-fold increase or decrease and a p-value $<0.01$ between PC3 cells with ETS-1 blockade compared to mock control were considered to indicate a statistically significant difference (Tables I and II).

The recently reported immunity-related genes that were found to be upregulated $>2$-fold in ETS- 1 blockade compared to the mock control are $C D 38$ and HDAC9 (Table I). Conversely, the genes that were found to be downregulated are $B C L 11 A$, CRISP3, DMBT1, FGR, NOS2 and SAA1 (Table II). 


\section{Discussion}

As knowledge of the immune defenses in $\mathrm{PCa}$ remains incomplete, we recently reported the identification of immunity-related genes in PCa, and suggested (based on bioinformatical analysis) that the genes are potentially regulated by members of the ETS family of transcription factors (7), which are known to be involved in translocations frequently found in $\mathrm{PCa}(8)$, and are reported to be essential for the regulation of immunity-related genes (9). More specifically, our analysis revealed that 31 out of the 37 immunity-related genes that we have identified have potential binding sites for the ETS family prototype, ETS-1 (7).

As a continuation of our recent study, in which we identified immunity-related genes in $\mathrm{PCa}$ (7), we investigated whether ETS-1 regulates these PCa immunity-related genes in PC3 cells. We specifically blocked ETS-1 in PC3 PCa cells (14) and assessed the effect of the blockade on the expression of the recently identified PCa immunity-related genes (7) using a comprehensive oligo gene expression microarray analysis.

Our results reveal that out of the $31 \mathrm{PCa}$ immunity-related genes with potential ETS-1 binding sites within their promoter regions, the expression of 8 genes was found to be significantly up- or downregulated in PC3 cells with ETS-1 blockade compared to mock control (Tables I and II).

The PCa immunity related-genes $C D 38$ and $H D A C 9$ were found to be upregulated more than 2-fold in PC3 cells with ETS-1 blockade compared to the mock control (Table I), whereas BCL11A, CRISP3, DMBT1, FGR, NOS2 and SAAI were found to be downregulated (Table II).

The significant up- or downregulation of these 8 genes in PC3 cells with ETS- 1 blockade compared to mock control cells indicate that ETS-1 regulates these immunity-related genes directly or indirectly. These results suggest that ETS-1 represses the expression of the immunity-related genes $C D 38$ and $H D A C 9$, while activating the expression of $B C L 11 A$, CRISP3, DMBT1, FGR, NOS2 and SAA1 in PC3 PCa cells. Besides the roles of these genes in the immune response, several have been reported to be involved in various types of cancer, including PCa (17-28). However, the precise roles that these genes may play in the network of cellular and molecular mechanisms of antitumor immunology according to current models is not always clear from the literature. Briefly, decreased levels of CD38 have been reported in prostate adenocarcinoma cells (17). A high HDAC9 expression has been found in primary medulloblastoma samples and reported to be significantly associated with poor survival (18). In addition, a high expression of HDAC9 has also been found to be associated with poor prognosis in childhood acute lymphoblastic leukemia (19). BCL11A has been reported to be involved in lymphoid malignancies (20). CRISP3 is stated to be overexpressed in PCa (21) and suggested to be an independent predictor of recurrence following radical prostatectomy for localized PCa (22). DMBT1 has been reported to be involved in the carcinogenesis of the malignant brain tumors medulloblastoma and glibolastoma multiforme (23), and the DMBT1 gene has also been stated to be highly unstable in cancer, and to be involved in the immune defense (24). $F G R$ has been suggested to play a significant role in ovarian carcinoma cancer growth (25). NOS2 has been reported to be implicated and correlated with various prostatic diseases including PCa (26). Finally, SAAl has been found to be highly expressed in lung cancer tissue (27) and over-expressed in ovarian carcinoma tissues (28).

In correlating our results from the PC3 cells with the recent findings from the $\mathrm{PCa}$ tissues (7), we found that the PCa immunity related genes CRISP3 and FGR, which were reported to be upregulated in $\mathrm{PCa}$ glands compared to normal glands (7), are downregulated in PC3 cells with ETS-1 blockade compared to the mock control (Table II). Conversely, $C D 38$, which was reported to be downregulated in prostate carcinoma glands compared to normal glands (7), is found to be upregulated in PC3 cells with ETS-1 blockade compared to the mock control (Table I). These observations suggest that ETS-1 may be involved in the activation of the immunity-related genes CRISP3 and FGR, and the repression of $C D 38$ in $\mathrm{PCa}$.

Of note, correlating results from $\mathrm{PCa}$ cell lines such as PC3 cells, with the findings from primary PCa tissues should be interpreted with caution. A major issue is that the three most commonly used cell culture models of PCa, DU145, PC3 and LNCaP, are metastatic cell lines derived from brain, advanced androgen-independent bone and supraclavicular lymph node metastasis, respectively. Although these cell lines are useful cell culture models for $\mathrm{PCa}$, the fact that they were derived from metastatic sites, rather than primary $\mathrm{PCa}$ tumors, makes correlating findings from $\mathrm{PCa}$ cell lines with primary $\mathrm{PCa}$ tumor tissues a challenge, as gene changes may occur during tumor progression. Therefore, primary PCa cell lines would be the optimal choice; however, such cell lines are difficult to culture and maintain for a long period of time.

We report that 8 of the recently identified $\mathrm{PCa}$ immunity-related genes are regulated by ETS-1 in PC3 PCa cells. These findings provide insights into the regulation of immunity-related genes in $\mathrm{PCa}$, and emphasize the importance of ETS-1 in PCa immunology.

In conclusion, this study investigated the regulation of immunity-related genes, recently identified from PCa tissues in the in vitro PCa cell line model, PC3 cells. Our results have shown that ETS-1 is involved in the activation and repression of immunity-related genes in PCa. To determine the precise molecular mechanisms of ETS-1 regulation of these genes requires further investigation, including DNA binding and co-transfection assays. However, these findings provide further insight into the molecular aspects of PCa immunology, and emphasize the importance of ETS-1 in PCa immunology.

\section{Acknowledgements}

We are grateful to the Deutsche Forschungsgemeinschaft (DFG); German Research Association, grant no. WE 1104/11-1) and the Deutsche Krebshilfe (German Cancer Aid, grant no. 107827) for financial support.

\section{References}

1. Kumar-Sinha C, Tomlins SA and Chinnaiyan AM: Recurrent gene fusions in prostate cancer. Nature Rev 8: 497-511, 2008.

2. Hsu DS, Kim MK, Balakumaran BS, et al: Immune signatures predict prognosis in localized cancer. Cancer Invest 28: 765-773, 2010 . 
3. Lin WW and Karin M: A cytokine-mediated link between innate immunity, inflammation, and cancer. J Clin Invest 117: 1175-1183, 2007.

4. Shimura S, Yang G, Ebara S, Wheeler TM, Frolov A and Thompson TC: Reduced infiltration of tumor-associated macrophages in human prostate cancer: association with cancer progression. Cancer Res 60: 5857-5861, 2000.

5. Vesalainen S, Lipponen P, Talja M and Syrjanen K: Histological grade, perineural infiltration, tumour-infiltrating lymphocytes and apoptosis as determinants of long-term prognosis in prostatic adenocarcinoma. Eur J Cancer 30A: 1797-1803, 1994.

6. Rajarubendra N, Lawrentschuk N, Bolton DM, Klotz L and Davis ID: Prostate cancer immunology - an update for Urologists. BJU Int 107: 1046-1051, 2010.

7. Shaikhibrahim Z LA, Ellinger J, Rogenhofer S, Buettner R and Wernert N: Identification of immunity-related genes in prostate cancer and potential role of the ETS family of transcription factors in their regulation. Int J Mol Med 28: 799-807, 2011.

8. Tomlins SA, Rhodes DR, Perner S, et al: Recurrent fusion of TMPRSS2 and ETS transcription factor genes in prostate cancer. Science 310: 644-648, 2005.

9. Gallant S and Gilkeson G: ETS transcription factors and regulation of immunity. Arch Immunol Ther Exp (Warsz) 54: 149-163, 2006

10. Alipov G, Nakayama T, Ito M, et al: Overexpression of Ets-1 proto-oncogene in latent and clinical prostatic carcinomas. Histopathology 46: 202-208, 2005.

11. Rothhammer T, Hahne JC, Florin A, et al: The Ets-1 transcription factor is involved in the development and invasion of malignant melanoma. Cell Mol Life Sci 61: 118-128, 2004.

12. Hahne JC, Okuducu AF, Kaminski A, Florin A, Soncin F and Wernert N: Ets-1 expression promotes epithelial cell transformation by inducing migration, invasion and anchorageindependent growth. Oncogene 24: 5384-5388, 2005.

13. Sahin A, Vercamer C, Kaminski A, et al: Dominant-negative inhibition of Ets 1 suppresses tumor growth, invasion and migration in rat C6 glioma cells and reveals differentially expressed Ets 1 target genes. Int J Oncol 34: 377-389, 2009.

14. Shaikhibrahim Z, Langer B, Lindstrot A, et al: Ets-1 is implicated in the regulation of androgen co-regulator FHL2 and reveals specificity for migration, but not invasion, of $\mathrm{PC} 3$ prostate cancer cells. Oncol Rep 25: 1125-1129, 2011.

15. Shaikhibrahim Z, Lindstrot A, Langer B, Buettner R and Wernert N: Comprehensive gene expression microarray analysis of Ets-1 blockade in PC3 prostate cancer cells and correlations with prostate cancer tissues: Insights into genes involved in the metastatic cascade. Int J Mol Med 27: 811-819, 2011.
16. Chen $\mathrm{CA}$ and Okayama $\mathrm{H}$ : Calcium phosphate-mediated gene transfer: a highly efficient transfection system for stably transforming cells with plasmid DNA. BioTechniques 6: 632-638, 1988.

17. Liu AY, Roudier MP and True LD: Heterogeneity in primary and metastatic prostate cancer as defined by cell surface $C D$ profile. Am J Pathol 165: 1543-1556, 2004.

18. Milde T, Oehme I, Korshunov A, et al: HDAC5 and HDAC9 in medulloblastoma: novel markers for risk stratification and role in tumor cell growth. Clin Cancer Res 16: 3240-3252, 2010

19. Moreno DA, Scrideli CA, Cortez MA, et al: Differential expression of HDAC3, HDAC7 and HDAC9 is associated with prognosis and survival in childhood acute lymphoblastic leukaemia. Br J Haematol 150: 665-673, 2010.

20. Satterwhite E, Sonoki T, Willis TG, et al: The BCL11 gene family: involvement of BCL11A in lymphoid malignancies. Blood 98: 3413-3420, 2001.

21. Kosari F, Asmann YW, Cheville JC and Vasmatzis G: Cysteine-rich secretory protein-3: a potential biomarker for prostate cancer. Cancer Epidemiol Biomarkers Prev 11: 1419-1426, 2002.

22. Bjartell AS, Al-Ahmadie H, Serio AM, et al: Association of cysteine-rich secretory protein 3 and beta-microseminoprotein with outcome after radical prostatectomy. Clin Cancer Res 13: 4130-4138, 2007.

23. Mollenhauer J, Wiemann S, Scheurlen W, et al: DMBT1, a new member of the SRCR superfamily, on chromosome 10q25.3-26.1 is deleted in malignant brain tumours. Nat Genet 17: 32-39, 1997.

24. Mollenhauer J, Herbertz S, Holmskov U, et al: DMBT1 encodes a protein involved in the immune defense and in epithelial differentiation and is highly unstable in cancer. Cancer Res 60: 1704-1710, 2000

25. Kim HS, Han HD, Armaiz-Pena GN, et al: Functional roles of Src and Fgr in ovarian carcinoma. Clin Cancer Res 17: 1713-1721, 2011.

26. Yoo KH, Kim SK, Chung JH and Chang SG: Nitric oxide synthase 2 gene polymorphisms are associated with prostatic volume in Korean men with benign prostatic hyperplasia. Asian J Androl 12: 690-696, 2010.

27. Sung HJ, Ahn JM, Yoon YH, et al: Identification and validation of SAA as a potential lung cancer biomarker and its involvement in metastatic pathogenesis of lung cancer. J Proteome Res 10: 1383-1395, 2011.

28. Urieli-Shoval S, Finci-Yeheskel Z, Dishon S, et al: Expression of serum amyloid a in human ovarian epithelial tumors: implication for a role in ovarian tumorigenesis. J Histochem Cytochem 58: 1015-1023, 2010. 